\title{
The experience of the use of Community Treatment Orders following Recovery-Oriented Practice training
}

Vrinda Edan, ${ }^{1,2}$ Lisa Brophy, ${ }^{2,3,7}$ Penelope June Weller, ${ }^{4}$ Ellie Fossey, ${ }^{5}$ Graham Meadows, ${ }^{1,2,6}$

${ }^{1}$ Southern Synergy, Department of Psychiatry, Monash University, 126 Cleeland St, Dandenong, VIC, Australia

${ }^{2}$ Melbourne School of Population and Global Health, University of Melbourne, Parkville, VIC, Australia

${ }^{3}$ Mind Australia, Heidelberg, VIC, Australia

${ }^{4}$ Graduate School of Business and Law, RMIT University, Melbourne, VIC, Australia

${ }^{5}$ School of Primary and Allied Health Care, Monash University Peninsula Campus, Frankston, VIC, Australia

${ }^{6}$ Monash Health, Melbourne, VIC, Australia

${ }^{7}$ School of Health Sciences, La Trobe University, Melbourne, VIC, Australia

Corresponding Author: Vrinda Edan, v.edan@unimelb.edu.au

\section{Abstract}

States across Australia are changing and adapting policy and laws to deliver mental health services using principles of personal recovery. Yet, the use of Community Treatment Orders (CTOs) remains high in apparent contradiction with this change. As part of the PULSAR trial investigating the outcomes of recovery oriented practice (ROP) training in primary and secondary care services within Metropolitan Melbourne, Victoria, a qualitative study was undertaken to explore the intersection between implementing ROP and working with consumers on CTOs. In-depth interviews were undertaken with consumers with experience being on CTOs and staff of secondary care services, and inductively analysed to identify themes. For consumers, being on a СTO meant lacking choice and control, an emphasis on medication, fear of the threat of hospitalisation, an absence of recovery oriented practice, and staying supported. For staff, recovery oriented practice in the presence of CTOs is challenging, with CTOs being seen to be a primary way to manage risk. Staff supported recovery as a practice, but identified a lack of organisational 'buy in' by services. The findings of this small scale study, embedded in a much larger study about ROP, support other literature that identifies implementing ROP in services that use CTOs as potentially problematic; and that ROP can enhance both consumers and staff experiences of services but, without systemic change, there may not be a significant shift in the use of CTOs, while CTOs also inhibit uptake of ROP.

\section{Keywords}

Recovery, 
Recovery Oriented Practice

Community Treatment Orders

Qualitative

\section{Introduction}

Mental health services in Australia are increasingly moving towards using principles of recovery oriented practice, with strong support from federal and state policy and legislation

(Commonwealth of Australia, 2013). Recovery oriented practice recognises that recovery may be different for each consumer, supports people to define their own goals for recovery and maximises self-determination and self-management of their mental health (Commonwealth of Australia, 2013, p. 15). Yet, in mental health services, Community Treatment Orders (CTO) are used to enforce treatment in the community, outside of hospital inpatient facilities, even though an international group of authors who have been leading the shift to recovery- oriented practice have concluded that compulsory treatment is incompatible with the key principles of personal recovery (Slade et al. 2014).

One of the main challenges of using CTOs in a recovery oriented service is that many recovery oriented practice frameworks (Commonwealth of Australia, 2013; Oades, et al., 2005; Leamy , at.al., 2011; Department of Health, 2011; Slade, 2009) highlight the importance of empowerment of consumers to make their own decisions, including but not limited to decisions about treatment. Utilising legal mechanisms, such as CTOs, are contradictory to this principle, but the policy documents tend to be silent on how to manage this contradiction (Light, Kerridge, Ryan \& Robertson, 2012a). Mary O'Hagan (2012), a prominent consumer advocate, has questioned the place of 'legal coercion' in a mental health system that purports to be recovery focused and challenges the ability of services to genuinely apply recovery oriented principles when they are utilising legally authorised or sanctioned compulsory practices, such as CTOs. However, other than concerns about lack of empowerment and autonomy, such as those raised by O'Hagan (2012), and despite these being core concepts related to recovery, the recoveryoriented practice (ROP) literature provides very little guidance regarding the relevance and implications of ROP to CTOs.

In Australia, rates of CTO use are relatively high compared to international rates (Light, Kerridge, Ryan \& Robertson, 2012b). The state of Victoria, in particular, has a long and extensive history of using them, with over 5,000 people on Community Treatment Orders at any given time or more 
than $25 \%$ of clients of community mental health services (Light, et al., 2012 b) Furthermore, Light et al. (2012 b) reported that between 2005 and 2011 rates in Victoria increased from 55 to 98.8 people on CTOs per 100,000 population. The effectiveness of CTOs is also highly debated (O'Brien, McKenna \& Kydd, 2008; Rugkasa, 2016), so that further understanding of the experience of consumers in relation to the impact of CTOs and utilisation of recovery oriented practice is needed. This paper presents the findings from a qualitative study that sought to explore these issues.

\section{Background to the study}

A systematic review of qualitative studies (Corring, O'Reilly \& Sommerdyk, 2017), a literature review (O'Brien, McKenna \& Kydd, 2008) and a recently updated Cochrane review of randomised controlled trails (Kisley, Campbell \& O'Reilly, 2017) all provide useful background information for this study. In their review of literature on CTOs from the previous two decades, O'Brien et al. (2008) described the types of orders in use and highlighted that studies had found both strong opposition to CTOs and some perceived benefits, with stronger support for CTOs among clinicians than service users. Based on a recent systematic review of consumer views and experiences, Corring et al. (2017) noted that despite some previous researchers have claimed there is a limited understanding of consumer experiences of CTOs, "we actually know a lot about the views and experiences of subjects of CTOs." ( $p$ 78). These authors reviewed 22 qualitative studies that explored the experiences of 581 participants from 7 countries around the world. They identified 10 common themes across the studies, with 3 themes having the strongest presence across all studies: feelings of being coerced and controlled, medication adherence seen as the main reason for CTOs, and CTOs seen as providing a safety net (Corring et al., 2017, p 76).

A Cochrane review of randomised controlled trials (RCTs) by Kisley et al (2017) investigated evidence for the effectiveness of CTOs. These authors drew the conclusion that the use of CTOs showed "no clear difference in service use, social functioning or quality of life compared with voluntary care or brief supervised discharge" $(p 1)$. They also noted that their review identified only three low to medium quality studies, with small numbers of participants and some blinding bias issues, so that it was difficult to draw definitive conclusions and that more research is needed. More recent research in New South Wales, Australia, has obtained further evidence about positive outcomes of CTOs in relation to readmission rates and increasing community care (Harris et al., 2018). However, as Ryan (2018) concludes, this evidence remains modest and reduced readmission rates may be the result of the increased contact with community based services associated with use of CTOs, or people on CTOs being prioritised for service delivery. 
Overall, this CTO literature tends to focus either on the experience of being on orders or on clinical outcomes, with minimal discussion about how ROP might change or contribute to practice with people on CTOs, or how this might impact the experience of being on a CTO. This study sought to explore these issues within the context of a trial of ROP training for staff in community mental health services in Victoria.

\section{PULSAR Intervention}

Mental health law and policy, and most service provision, is a state-based responsibility in Australia. In 2011, the Australian State of Victoria released 'A Framework for Recovery Oriented Practice' (Department of Health, 2011) and in 2014, the new Victorian Mental Health Act was implemented, which refers to recovery in both its objectives and principles while continuing to retain the use of compulsory powers, including CTOs (Department of Health Victoria, n.d.). During this time, the Victorian Government funded a large translational research project in Metropolitan Melbourne known as PULSAR (Principles Uniting Local Services Assisting Recovery) (Meadows et al., 2019). It utilised a mixed-methods stepped-wedge cluster randomized control trial design to evaluate whether adults accessing secondary and primary care mental health services, in which staff received purposely developed recovery-oriented practice training, reported superior recovery outcomes compared to those accessing services where staff had not received this training (Enticott et al., 2016; Shawyer et al., 2017). Several nested qualitative studies were also undertaken to understand the experiences of consumers and staff from the services where this training was provided. The PULSAR project engaged with lived experience from the outset with a consumer academic (VE) as an investigator on the project, who codesigned and delivered the training intervention and facilitated a Lived Experience Advisory Panel that contributed to the project throughout.

The PULSAR staff training was based on the REFOCUS intervention, originally developed and trialled in the UK (Slade, et al., 2011) and adapted by the PULSAR team, in collaboration with the REFOCUS investigators, to improve its relevance to the service environment in Victoria (Meadows et al., 2019). Specifically, given the substantial difference in CTO usage between Australia and the UK, an expert in mental health law and its implications for practice joined the PULSAR investigator team (PW). The staff training materials were adapted to include a section on CTOs and risk (Shawyer et al., 2017). The PULSAR intervention for staff of community mental health services consisted of two days of training. All training was co-facilitated by a clinician from the sector and an experienced consumer trainer. The training included didactic teaching 
and experiential learning, supported with a manual, tools and resources. Key principles from the REFOCUS intervention adopted for PULSAR included the CHIME framework, Connection, Hope, Identity, Meaning and Purpose and Empowerment; three working practices, 1) Working with strengths, 2) Consumers values and treatment preferences and 3) Goal Striving; and the use of a coaching approach (Bird, Leamy, Le Boutillier, Williams \& Slade, 2011). Staff were given opportunities within the training to explore the application of these principles and working practices to their own practice. An external expert in coaching also worked with staff on moving from an approach of being clinician as expert to working with people as joint experts. Following the training, all staff were provided with an opportunity to participate in team-based monthly sessions called PALS (PULSAR active learning sessions), designed to support staff implementation of the recovery-oriented practice training.

\section{Design and Methods}

Nested within the PULSAR trial described above, a qualitative study was designed to explore the relevance of ROP to people on CTOs and the staff working with them. It was conducted in 2017 and involved semi structured interviews with consumers and staff from community mental health services where the PULSAR trial occurred. Human research and ethics approval was granted through Monash Health (Ref No. 14427B). Recruitment of consumer participants was via a letter directly to consumers who had participated in the PULSAR trial and had consented to contact about further research. Only consumers identified as having been on a CTO in the previous 12 months were contacted. 43 letters were posted, with 11 consent forms being returned. Six participants could be contacted and were interviewed. The consumer investigator on the PULSAR team undertook the six consumer interviews, with the majority (5) being interviewed in person and one consumer participated by telephone. Staff recruitment occurred via email invitation to all staff of teams that participated in the PULSAR training intervention. Three staff gave consent to participate, and were interviewed by telephone. These interviews were conducted by a member of the PULSAR research team with a health professional background. Semi-structured interview guides for the consumer and staff interviews were developed by the PULSAR Qualitative Research Steering group, informed by members' lived expertise and knowledge of the literature on CTOs, personal recovery and recovery-oriented practices. All interviews were recorded, transcribed, and participants were offered an opportunity to review the transcripts, so as to check for errors or amend their transcripts if they wished, prior to analysis. 


\section{Data analysis}

Data analysis was undertaken by researchers including the consumer investigator. Consumer and staff responses were coded, and a thematic analysis followed. NVivo (2010) software was used to undertake the data analysis. An inductive approach was used, so as not to impose a theoretical framework on the analysis (Thomas, 2006). Initially, VE coded the consumer interviews, cross checking this coding with LB, while LB coded the staff interviews and cross checked her coding with VE. Patterns and themes across the responses from each participant were identified, and then summaries of the themes were created for the consumer and staff interviews.

\section{Description of participants}

Of the 6 consumer participants, 5 were female and their average age was 41.6 (range $36-58$ ). The participants identified as Australian (3) Malaysian (2) and Italian (1). Their length of service use varied from 5 to 42 years with an average of 15.3 years. All the participants identified using hospital based or clinical services and community managed mental health services.

Of the three staff who participated in these interviews, two were from the clinical service and one from a community managed mental health service. They were all female, their average age was 38.1 years (range $29-55$ years), and their average length of experience of working in mental health services was 14 years (range 11 to 28 years).

\section{Findings}

The themes identified from the consumer interviews about being on a CTO, and from staff about working with people on CTOs are summarised in Table 1. Each theme is then described, accompanied by illustrative quotes from participant interviews.

Table 1: Summary of themes for consumers and staff.

\begin{tabular}{|c|r|}
\hline Consumer themes about being on a CTO & $\begin{array}{r}\text { Staff themes about working with people on } \\
\text { CTOs }\end{array}$ \\
\hline $\begin{array}{l}\text { 1. Lacking choice and control } \\
\text { 2. Emphasis on medication }\end{array}$ & $\begin{array}{l}\text { 1. Recovery Oriented Practice in the } \\
\text { presence of CTOs is challenging }\end{array}$ \\
3. The threat of hospitalisation & $\begin{array}{l}\text { 2. CTOs are a way to manage risk } \\
\text { 4. Absence of recovery oriented }\end{array}$ \\
$\begin{array}{l}\text { 3. Lack of focus on recovery as a practice } \\
\text { 5. Staying supported }\end{array}$ & 4. Organisational 'buy in' lacking \\
\hline
\end{tabular}




\section{Consumer Themes}

Five main themes were identified in the analysis of the consumer interviews in relation to being on a CTO as follows:

i. Lacking choice and control,

ii. Emphasis on medication,

iii. The threat of hospitalisation,

iv. Absence of recovery oriented practice, and

v. Staying supported.

\subsection{Lacking choice and Control}

All six consumer participants shared a sense of the lack of choice and control that meant being on a CTO was experienced as burdensome or holding them back:

Well it's just draining and it saps me of energy - I just feel weak and sick all the time - like I've got a millstone like a big burden weighing down upon me - I just feel like it's total - but I've been through it before and I've been through worse so I feel like I can recover again but the community treatment order is not helping me at all. I would rather get a voluntary treatment order. (C1)

It would be better if I can take it [medication] on my own, instead of being pushed (C2)

This lack of control contributed not only to a reliance on services or other people, but also to a sense of being imposed upon by services:

[being on a CTO] lowers your independence levels because you know you haven't got real control of things (C3)

Yeah not having someone coming at 8 o'clock at night to give you medication you know you feel like you're a child (C2)

\subsection{Emphasis on medication}

All six consumer participants spoke about the emphasis on medication and a consistent message from the service that consumers must take the medication, or they will be sent back to hospital: Mostly about medication - take the medication I won't go to hospital again (C4) 
psychiatry doesn't do nothing, psychiatry just makes you ill and makes you weak and sick and here's your medicine - by luck it might be good, by luck it might be bad (C1)

This focus on medication was accompanied by a sense of surveillance, or being kept an eye on, which one participant described as being 'strict' for those on CTOs:

Well they keep an eye on you, they make sure that I take my medication, so I don't go back in hospital (C2)

Well I think on a CTO they were very strict, they really really look after your medication (C5)

\subsection{The threat of hospitalisation}

A number of the consumers spoke of the threat of hospitalisation if they did not take medication, and that the reason that they had been put them on a СТO by the service was to ensure that they could be taken back to hospital if they stopped taking medication:

Honest... bad but I am scared like I said if I say that I am hearing the voices again, or anything like that, they will put me in hospital again and I don't want to go (C2)

I think I am [on a CTO] I think that if I stop taking my medication they put me in hospital (C6)

Consumer often felt that they were only given two choices, hospital or CTO, without getting the appropriate supports to avoid either.

It actually makes me feel, I don't know - scared because I have been getting an ultimatum, whether to go one [hospital] or the other [CTO] you know if I keep it up and in one way I wasn't getting the support [to stay out of hospital] when they were saying that. I was just being told - there might have been support to their eyes but to me it wasn't; it was just like being told off (C3)

\subsection{Absence of recovery-oriented practice}

Some consumer participants spoke highly of individual staff members and, when prompted, they recalled some discussion of recovery principles such as working towards goals. However, most consumer participants had not had a conversation with clinical staff that they could identify as recovery focussed: 
there is no real plan to get me off the community treatment order and get me back into good health (C1)

Interviewer: ... whether recovery and talking to people about things like connecting with the community, about hope and about...

They don't talk to me about that (C2)

There were however signs of change within the services that consumer participants had noticed:

The way they're doing things is much different than before. Before it was - they used to talk to you but didn't ask questions, they used to tell you what you had to do and that was it - see you later (C2) It has improved though ... my case manager is on the phone just before I got here saying they've approved funding for a bed for me - so that's come through, so I would say that's on the positive side. It has improved over the last 6 months (C1)

\subsection{Staying supported}

Overall, the sense of lacking choice and control, the emphasis placed on medication and the threat of hospitalisation were more dominant concerns in the consumer interviews but, on balance, having supports was also an important element of being on a CTO for some consumer participants. Thus, several consumer participants made comments regarding the value of staff assisting with access to other services:

Having a case manager helps me connect with the supports that I need - because whatever I need I actually know what I want but I do not know how to get it (C5) they help me get on in touch with medical service, that's providing support (C1) In addition, for others, being on a CTO could ensure access to services that might be difficult to attend for other reasons, such as transport issues, as this participant described:

Makes a difference - it's good because I can't drive it helps me because I only wait for the nurse to come to give my regular injection I don't have to have the trouble to go there to the clinic, they give me convenience because I can't drive (C4) 


\section{Staff Themes}

All three staff participants identified that working with people on CTOs was a significant part of their work, albeit that there were fluctuations in the number of people on a СТO with whom they worked at any given time. They also all spoke about the importance of recovery in their practice and believed that the PULSAR training augmented their knowledge in the area, with one staff participant indicating that the training had strengthened a move towards co-design in their service.

Four consistent themes were identified across the staff interviews in relation to working with people on CTOs, as follows:

i. Recovery Oriented Practice in the presence of CTOs is challenging

ii. CTOs are a way to manage risk

iii. Lack of focus on recovery as a practice

iv. Organisational 'buy in' lacking

\subsection{Recovery Oriented Practice in the presence of CTOs is challenging}

All three staff spoke about working from a recovery focus, but they also spoke about the challenges of working with consumers on a CTO and practising in a recovery oriented way. Central to the principles of ROP is that of choice and control but within a service environment that also has a strong focus on risk management, supporting a consumer's choice and control can be very difficult:

allowing people the freedom to make their own choices and decisions, despite the risks is challenging on a CTO (S1)

that people's lives are not their own, and that they don't have the right to make risky choices, in the same way that the rest of us do (S2)

\subsection{CTOs are a way to manage risk:}

The predominant view of staff was that CTOs are a way to manage risk, rather than to support or promote recovery. Nevertheless, there was also reluctant support for CTOs, at least selectively for some consumers for whom staff could see some benefits:

because a CTO isn't about recovery, a CTO is about risk management (S2)

A big example for me would be someone who ... he'll be on a CTO forever, and I think that he is someone who does need to be on a CTO, and I don't say that lightly. (S2) 
For some of our clients they have reported that it's a protective factor, being on a CTO, for their own personal recovery, being on it has a bit of a safety net, (S1)

In this context, staff viewed a significant part of their role as that of advocacy, and that the PULSAR training intervention has supported them in this role:

As much as I hate to say it, that's kind of what you have to do in a lot of ways, stand up against risk aversion and promote dignity of risk and taking chances and giving people opportunities. I guess it (PULSAR) empowered me to really take that on in a more real way (S2)

\subsection{Support for recovery as a practice}

All three staff spoke about working from a recovery focus with consumers on CTOs. For staff who already felt that the principles of ROP were the 'cornerstone' of their practice, striving to practice in a way that supports dignity of risk and having different kinds of conversations with consumers were both important and rewarding elements of working with consumers in a recovery - oriented way:

What struck me most about PULSAR? I really, really liked it. I guess for me it backed up what I already believe in and strive to do in my practice. It sort of, like none of it was new to me ... but the whole concept of recovery oriented practice and dignity of risk and all of those things are something that I'm very passionate about and have been striving to work within for my entire professional career anyway. (S2)

The PULSAR recovery training also provided an opportunity to change the way they worked with consumers, including those on CTOs:

So that's been really lovely just to reframe the way that I think when I work with clients. And the way that I then get to talk to them about their goals and you know isn't it lovely having these goals about you know it's striving that's the most important. (S1)

Well look certainly initially you know, like after we'd done it I can remember finding myself in a situation and I thought ooh now I need to step back and go that way around it (S3) 


\subsection{Organisational 'buy in' lacking}

Of importance to two interviewed staff was the lack of organisational change. With a continuing focus on risk management, in which CTOs play a significant part, and with a lack of changes to paperwork and administrative requirements, maintaining a focus on recovery and implementing ROP in a systemic way was proving difficult for these staff:

You always find with any change management, if there's a change or a different way of needing to think, you're not going to get everyone on board, unfortunately that's what happens. (S1)

The other (issue) is our forms that we use at (service) are not very recovery orientated. (S1)

I would like to be more, even more deliberate I guess, because you really can get lost in being drowned with all of this paperwork and all of these administrative requirements. So you can definitely lose sight of these more important things. And I do wish I had been using those [ROP] tools more deliberately (S2)

Nevertheless, these two staff also indicated that there were signs of shifts within their own practice, and the service where they worked, towards promoting greater consumer choice and control:

I do think there's been a change in attitude around staff allowing clients to make more decisions for themselves. So, I think that as a result of the PULSAR, staff are more willing to give the clients that choice if they want to do something that we consider to be risky. I think we're better at doing that (S1)

I guess going through the training was a really nice, it was empowering, it helped me to see that no I do have, I do have quite a lot of power in my role, and I can use that to advocate for people more than I was (S2)

\section{Discussion}

The overall findings of this small study are consistent with the conclusions of Corring et al.'s (2017) systematic review. Both indicate that consumers subject to CTOs report experiences of coercion, lack of choice, that services focus on medication as the primary treatment, and that medication adherence is the main reason for a person being placed on a CTO. Given that one of the principles of recovery-oriented practice (ROP) is that of autonomy, it becomes apparent that 
implementing ROP is very problematic in an environment that not only supports the use of CTOs but also has the highest use of them (Light et al., 2012 a; Light et al., 2012 b). This is also highlighted by staff who acknowledged that СТO use is about the management of risk. Watson et al. (2014) argue that the use of force, such as CTOs, is a failure of the system and that "it is avoidable and can be reduced or eliminated through changing attitudes, practices, and environments of care" ( $p 530$ ). While the focus on risk continues in this way, the implementation of ROP across services will remain difficult to achieve. Similarly, both staff and consumers spoke about how CTOs enabled ongoing treatment as a 'safety net' and a way to access care. This supports the view that an unintended consequence of CTOs is that they assist in the management of systemic barriers to continuity of care. Again, this appears to suggest that CTOs are a response to service system failures, rather than individual characteristics of consumers. Corring et al.'s (2017) review also reported studies in which consumers identified support systems available to them while they were on CTOs. Leadership and management in services therefore need to address both the emphasis placed on risk, and the lack of continuity and individualisation of care in services, if they are genuinely planning to support ROP.

Both consumer and staff participants spoke about the lack of use of recovery-oriented language and tools in services. While some staff stated that they used the principles of recovery, they did not use ROP tools on the grounds that there was already so much paperwork to do. For consumers, the lack of use of ROP tools and language meant that they are less aware of any change in clinicians' practice. Nevertheless, the staff interviews suggested a genuine desire on behalf of staff for change, with examples of how some have facilitated changes in policy and practice within their service. At the same time, other staff expressed feeling powerless to make change happen and were waiting for 'the service' to make the changes necessary to support them to implement ROP. This highlights the importance of organisational support to effect change in practice, as also noted in previous ROP studies (Le Boutillier et al., 2015).

\section{Conclusion}

The findings of this study are consistent with evidence that CTOs have an impact on consumers' experiences of service use and staff experiences of working with people on CTOs. The experience of coercion by people on CTOs also aligns with staff views that CTOs are used as a risk management tool. There is however evidence of the benefit of ROP training for staff, both in rethinking their own practice with people on CTOs, and in being able to implement changes in the services where they work. Although these changes in practice were less evident to consumers, they indicate that there is value in providing ROP training and practice support 
within services that use CTOs and have goals of supporting practices that may reduce the use of coercive interventions.

\section{Limitations}

This small qualitative study was located in a metropolitan area of Melbourne, Victoria, and limited to community mental health services in which staff had undertaken the PULSAR training intervention. Given the specificity of this context, the findings may be less applicable to other settings. Nevertheless, given the legislative framework for use of CTOs and the ROP policy framework (Department of Health, 2011) are consistent across the state of Victoria, similar consumer and staff experiences could be expected at least within services in Victoria.

The number of participants is small, in part because recruitment was limited to services where the PULSAR training had been delivered. In addition, a relatively small number of consumers subject to CTOs had consented to participate in PULSAR and were thus eligible to participate in this study.

There is some previous commentary about the inherent difficulty of researching consumers experiences of CTOs, with one explanation given for this being that people subjected to CTOs have a history of being "uncooperative in following-up with mental health professionals" (Corring et al., 2017, p78) and so this 'uncooperativeness' may contribute to them not participating in research activities. In addition, these authors comment that using staff to recruit people into research projects is a potential barrier. The PULSAR research team acknowledged this issue, commonly referred to as 'gate keeping' and accepted the argument that this practice is unethical (Sharkey et al., 2010). Hence, consumers were directly contacted by letter to minimise the risk of only recruiting consumers who were either satisfied or dissatisfied with the service that they were receiving. Indeed, a range of experiences of being on a СTO were reported in this study. For instance, while two participants appeared not to agree with the necessity of being on the CTO at all, two others identified benefits and appreciated the additional services available to them. Despite being a small study, several efforts were also made to enhance the credibility of the findings, including the involvement of a researcher with lived experience, triangulation of data from different sources, and peer debriefing between the two researchers who undertook the data analysis.

\section{Funding}

The PULSAR project was funded by the Victorian Government, Department of Health's Mental IIIness Research Fund (MIRF project \#18). The views, analyses, interpretations, and conclusions 
expressed in the article are those of the authors, not of the Victorian Department of Health \& Human Services.

\section{Bibliography}

Bird, V., Leamy, M., Le Boutillier, C. Williams, J. \& Slade, M. (2011). REFOCUS: Promoting recovery in community mental health services. London: Rethink

Commonwealth of Australia (2013). A national framework for recovery-oriented mental health services: Guide for practitioners and providers. Canberra: Commonwealth of Australia.

Corring, D., O'Reilly, R., \& Sommerdyk, C. (2017). A systematic review of the views and experiences of subjects of community treatment orders. International Journal of Law and Psychiatry, 52, 74-80.

Department of Health Victoria (n.d.). Mental Health Act 2014. Retrieved August 2018, from https://www2.health.vic.gov.au/mental-health/practice-and-service-quality/mental-health-act-2014

Department of Health. (2011). Framework for recovery-oriented practice. State of Victoria. Retrieved August 2018, from

https://www2.health.vic.gov.au/about/publications/policiesandguidelines/Framework-for-Recoveryoriented-Practice

Enticott, J. C., Shawyer, S., Brophy, L., Russell, G., Fossey , E., Inder , B., Mazza, D., Vasi, S., Weller, P., Wilson-Evered, E., Edan, V. \& Meadows , G. (2016). The PULSAR primary care protocol: a steppedwedge cluster randomized controlled trial to test a training intervention for general practitioners in recovery-oriented practice to optimize personal recovery in adult patients. BMC Psychiatry, 16:451. http://dx.doi.org/10.1186/s12888-016-1153-6.

Harris, A., Chen, W., Jones, S., Hulme, M., Burgess, P., \& Sara, G. (2018). Community treatment orders increase community care and delay readmission while in force: results from a large population based study. Australian and New Zealand Journal of Psychiatry, Published Online (27 Feb 2018). http://dx.doi.org/10.1177/0004867418758920

Kisley, S. R., Campbell, L. A., \& O'Reilly, R. (2017). Compulsory community and involuntary outpatient treatment for people with severe mental disorders. Cochrane Database of Systematic Reviews (3). http://dx.doi.org/10.1002/14651858.CD004408.pub5.

Le Boutillier, C., Chevalier, A., Lawrence, V., Leamy, M., Bird, V. J., Macpherson, R., Williams, J. \& Slade, M. (2015). Staff understanding of recovery-orientated mental health practice: a systematic review and narrative synthesis. Implementation Science, 10:87, 1-14.

http://dx.doi.org/10.1186/s13012-015-0275-4

Leamy, M., Bird, V., Le Boutillier , C., Williams , J., \& Slade, M. (2011, Dec). Conceptual framework for personal recovery in mental health: systematic review and narrative synthesis. British Journal of Psychiatry, 199(6), 445-52.

Light, E. M., Kerridge, I. H., Ryan, C. J., \& Robertson, M. D. (2012a). Out of sight, out of mind: making involuntary community treatment visible in the mental health system. Medical Journal of Australia, 196(9), 591-593.

Light, E., Kerridge, I., Ryan, C., \& Robertson, M. (2012b). Community Treatment orders in Australia: rates and patterns of use. Australasian Psychiatry, 20(6), 478-482. 
Meadows, G., Brophy, L., Shawyer, F., Endicott, J., Fossey, E., Weller, P., Wilson-Evered, E., Edan, V. \& Slade, M. (2019). REFOCUS-PULSAR recovery-oriented practice training in specialist mental health care: a stepped-wedge cluster randomised controlled trial. Lancet Psychiatry, 6(2), 103-114. http://dx.doi.org/10.1016/S2215-0366 (18)30429-2

NVivo qualitative data analysis Software. (2010). Version 10. QSR International Pty Ltd.

Oades, L., Deane, F., Crowe, T., Lambert, W. G., Kavanagh, D., \& Lloyd, C. (2005). Collaborative recovery: an integrative model for working with individuals who experience chronic and recurring mental illness. Australasian Psychiatry, 13(3), 279-284.

O'Brien, A. J., McKenna, B. G., \& Kydd, R. R. (2008). Compulsory community mental health treatment: Literature review. International Journal of Nursing Studies, 46, 1245-1255.

O'Hagan, M. (2012, Jan 17). Legal coercion: the elephant in the recovery room. Retrieved July 20, 2018, from Scottish Recovery Network: https://www.scottishrecovery.net/resource/legal-coercionthe-elephant-in-the-recovery-room/

Rugkasa, J. (2016). Effectiveness of Community Treatment Orders: The International Evidence. Canadian Journal of Psychiatry, 61(1), 15-24.

Ryan, C. (2018). Community treatment orders are (somewhat) effective: Their future in the context of rights-based mental health law. Australian and New Zealand Journal of Psychiatry, 53 (1), 11-12 http://dx.doi.org/10.1177/0004867418791300

Sharkey, K., Savulescu, J., Aranda, S., \& Schofield, P. (2010). Clinician gate-keeping in clinical research is not ethically defensible: an analysis. Journal of Medical Ethics, 36, 363-366.

http://dx.doi.org/10.1136/jme.2009.031716

Shawyer, F., Enticott , J., Brophy, L., Bruxner, A., Fossey, E., Inder, B., Julian, J., Kakuma, R., Weller, P., Wilson-Evered, E. Edan, V., Slade, M. \& Meadows, G. (2017). The PULSAR Specialist Care protocol: a stepped-wedge cluster randomized control trial of a training intervention for community mental health teams in recovery-oriented practice. BMC Psychiatry, 17:172 http://dx.doi.org/10.1186/s12888-017-1321-3

Slade, M. (2009). Personal Recovery and mental Illness. A guide for mental health professionals. Cambridge: Cambridge University Press.

Slade, M., Bird, V., Le Boutillier, C., Williams, J., McCrone, P. \& Leamy, M. (2011). REFOCUS Trial: protocol for a cluster randomised controlled trial of a pro-recovery intervention within community based mental health teams. BMC Psychiatry, 11:185. http://dx.doi.org/10.1186/1471-244X-11-185

Slade, M., Amering, M., Farkas, M., Hamilton, B., O'Hagan, M., Panther, G., Perkins, R., Shepherd, G., Tse, S. \& Whitley, R. (2014). Uses and abuses of recovery: Implementing recovery-oriented practices in mental health systems. World Psychiatry, 13, 12-20. http://doi.org/10.1002/wps.20084

Thomas, D. (2006). A general inductive approach for analyzing qualitative evaluation data. American Journal of Evaluation, 27(2), 237-246.

Thomas, D. R. (2003). A general inductive approach for qualitative data analysis: School of Population Health. New Zealand: University of Auckland. 
Watson, S., Thorburn, K., Everett, M. \& Fisher, K. R. (2014). Care without coercion - mental health rights, personal recovery and trauma informed care. Australian Journal of Social Issues, 49(4), 529549. 